International Journal of Pure and Applied Mathematics

Volume 93 No. 1 2014, 147-154

ISSN: 1311-8080 (printed version); ISSN: 1314-3395 (on-line version)

url: http://www.ijpam.eu

doi: http://dx.doi.org/10.12732/ijpam.v93i1.12

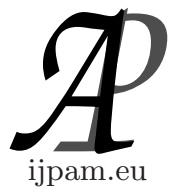

\title{
SOLVING PORTFOLIO OPTIMIZATION PROBLEMS WITH STRUCTURED PRODUCTS
}

\author{
Meena Baweja ${ }^{1}$, Ratnesh R. Saxena ${ }^{2}$ \\ ${ }^{1}$ Department of Mathematics \\ University of Delhi \\ Delhi 110007, INDIA \\ ${ }^{2}$ Department of Mathematics \\ Deen Dayal Upadhyay College \\ Delhi University, Delhi, INDIA
}

\begin{abstract}
This paper investigates how a portfolio based on structured products should be constructed and rebalanced to enhance the return potential. The focus lies on minimizing the risk of the fund. Due to possible intermediate payments from structured products, we have to deal with re-investment problem. The investor re-balances its portfolio at time of maturity of each structured product.
\end{abstract}

Key Words: conditional value-at-risk, structured products, capital-protected, portfolio optimization

\section{Introduction}

Portfolio optimization has come a long way from early fifties when Markowitz [3] seminal work introduces return/variance risk management framework. Current regulations for finance business formulate some of the risk management requirements in terms of percentiles of loss distributions. An upper percentile of loss distribution is called VaR (Value-at-Risk). VaR means the amount of money that expresses the maximum expected loss from an investment over a specific investment horizon for a given confidence level.It has great popularity

Received: January 19, 2014

(c) 2014 Academic Publications, Ltd.

$\S$ Correspondence author url: www.acadpubl.eu 
among banks, insurance companies and other financial institutions, but VaR (Value-a-Risk) does not give any information beyond this amount of money. Further, it has undesirable mathematical characteristics such as lack of subadditivity and convexity (Artzner et al. 1999) [2]. VaR is also difficult to optimize for discrete distributions when it is calculated using scenarios. The paper suggests to use, as a supplement to $\mathrm{VaR}$, another percentile risk measure which is called Conditional Value-at-Risk (CVaR). CVaR expresses the expected loss of an investment beyond its VaR value. In the year 2001, Rockafellar and Uryasev [5] discussed the subadditive and convex property of CVaR. Coherency of CVaR was proved by Rockafellar and Uryasev [7] in the year 2000. Acerbi et al. [1] also discussed the same property for CVaR. In this paper, we consider the aspect of structured product as it is safe investment that guarantees a minimum payoff at maturity and permits profiting from positive developments in the underlying markets. In other words here structured products are bought and hold until maturity. To offer advantages of diversification in structured product to small investors, the concept of a fund based on structured products emerges. We consider a portfolio having structured products which do not exactly match the investor's needs as they have maturity before the investor's horizon time. Due to the intermediate payments from structured products, the one-period (Martellini et al., 2005 [4]) problem gets a dynamic aspect. The first study conducted in the year 2009 on the subject, using a longer horizon time then the time to maturity for the structured product, was performed by Rolf Korn et al. [6]. We develop a multi-period model for optimizing a portfolio using the concept of structured products.

This paper is structured in the following way. Section 1 makes readers familiar with the concept of measures of risk, CVaR and structured products. Section 2 reveals how the portfolio choice problem is formulated by using modified Korn and Zeytun framework. Section 3 ends with the conclusion.

\subsection{Risk Measures}

In the process of measuring the risk, the selection of risk measure has a critical importance. In traditional portfolio theory, the risk of a portfolio is measured in terms of its variance. This approach is based on the assumption that the portfolio's return distribution is symmetric. Since the return distribution of a portfolio containing derivatives becomes non-symmetric, there is a need for another risk measures. For non-symmetric return distributions, VaR and CVaR are used as risk measures. VaR describes the predicted maximum loss with confidence level $\beta$ over a pre-specified period of time. CVaR is expectation of 
the loss when the loss exceeds or equals the VaR value. Let $L(w, y)$ be the loss associated with decision vector $w$ and random vector $y$ in $\mathbb{R}^{m}$. The probability of $L(w, y)$ not exceeding a threshold $\alpha$ is given by

$$
\phi(w, \alpha)=P(L(w, y)) \leq \alpha .
$$

The Value-at-Risk $\left(\operatorname{VaR}_{\beta}\right)$ is the loss with confidence level $\beta \in[0,1]$ defined by

$$
\begin{aligned}
& \operatorname{VaR}_{\beta}(L(w, y))=\min \{\alpha \in \mathbb{R}: \phi(w, \alpha) \geq \beta\}, \\
& \operatorname{VaR}_{\beta}(L(w, y))=\min \{\alpha \in \mathbb{R}:(P(L(w, y)) \leq \alpha) \geq \beta\},
\end{aligned}
$$

where

$$
L(w, y)=-R^{w}(y)
$$

here $R^{w}(y)$ is return associated with the decision vector $w$ and random vector $y$,

$$
\begin{gathered}
R^{w}(y)=\frac{\text { Final wealth }- \text { Initial wealth }}{\text { Initial wealth }} \\
\operatorname{CVaR}_{\beta}(L(w, y))=E\left(L(w, y) \mid L(w, y) \geq \operatorname{VaR}_{\beta}\right) .
\end{gathered}
$$

(Rockafellar et al. 2001, 2000 [5, 7]) have shown that

$$
\min _{w} \operatorname{CVaR}_{\beta}(L(w, y))=\min _{(w, \alpha)} F_{\beta}(w, \alpha)
$$

and

$$
F_{\beta}(w, \alpha)=\alpha+(1-\beta)^{-1} \int_{y \in \mathbb{R}^{m}}[L(w, y)-\alpha]^{+} P(y) d y,
$$

here $P(y)$ is density of probability distribution of $y$.

\subsection{Structured Products}

Structured products are synthetic investment instruments that are created to meet special needs for customers. In general these needs can not be met by the current market. Structured products are often constructed with low downside risk on one hand and the possibility of growth on the other hand. There are endless combinations of possible structured products. The most common set up for a structured product is the combination of zero-coupon bond and an option on an underlying. The bond and the option have same maturity time. Type of option varies widely and most often a European call option is used. The price of structured product on the date of issue is equal to the face value of the bond, which is set to be the current price of the underlying. The bond's face value is deterministic and payoff of the option is stochastic. To offer the advantages of diversification, a set of numerous assets is used in the portfolio which is very appealing especially if the investment is capital guaranteed. 


\subsection{Capital Guaranteed}

A product is capital guaranteed if the investor will receive atleast the invested amount at maturity. It is obvious that it is difficult to gain the property of capital-guarantee in combination with the possibility of high return. Moreover, it is clear that a portfolio based on many structured products will not hold the property of capital-guarantee, because a capital-guaranteed product has this property for a fixed investment period. Problems arises when the portfolio holds positions in products with maturity beyond the investment horizon, since all structured products can have a value of zero before maturity. Also if the structured products have maturity prior to the investment horizon's end, the issue of reinvestment of payoff arises. Thus for a portfolio having several structured products with different maturities, the terminology must be changed from capital guarantee to capital protection.

\section{Portfolio Optimization}

We assume that the investor can invest in $n$ assets where $n=u+m$, first $u$ assets are bonds $B_{1}, B_{2}, \ldots, B_{u}$ and last $m$ assets are structured products namely $S_{1}, S_{2}, \ldots, S_{m}$ with return vector $R_{T}=\left(R_{T}^{1}, R_{T}^{2}, \ldots, R_{T}^{n}\right)$ at horizon time $T$. Here $R_{T}^{i}$ is the return of asset $i, i=1,2, \ldots, n$.

Choosing the investment portfolio $w=\left(w_{1}, w_{2}, \ldots, w_{n}\right)$ where $w_{i}$ represents the weight of asset $i, i=1,2, \ldots, n$. The portfolio return at investment horizon $T$ is given by

$$
R_{T}^{w}=\sum_{i=1}^{n} w_{i} R_{T}^{i} .
$$

We are trying to maximize return with constraint on conditional Value-at-Risk that has upper bound ' $d$ '. The corresponding optimization problem is:

$$
\begin{aligned}
& \max _{w \in \mathbb{R}^{n}} E\left(R_{T}^{w}\right), \\
& \text { subject to } \\
& R_{T}^{w}=\sum_{i=1}^{n} w_{i} R_{T}^{i}, \\
& \sum_{i=1}^{n} w_{i}=1, \\
& w_{i} \geq 0, \quad i=1,2, \ldots, n,
\end{aligned}
$$




$$
\operatorname{CVaR}\left(-R_{T}^{w}\right) \leq d .
$$

\subsection{Linearization of CVaR}

Besides CVaR, the above problem is linear optimization problem is $w$. However, the CVaR seems to be highly non-linear in $w$. Fortunately in the year 2000, Rockafellar [7] discussed linearization procedure for CVaR by using function $F_{\beta}(w, \alpha)$. The integral given in equation (1.1) can be replaced by a summation, and in this case minimization of $F_{\beta}(w, \alpha)$ is equivalent to minimization of the linear expression

$$
\begin{aligned}
& \alpha+\frac{1}{N(1-\beta)} \sum_{k=1}^{N} z_{k}, \\
& \text { subject to } \\
& \qquad z_{k} \geq 0, \quad k=1,2, \ldots, N \\
& \quad L\left(w, y_{k}\right)-\alpha \leq z_{k}, \quad k=1,2, \ldots, N,
\end{aligned}
$$

where $z_{k}$ 's, $k=(1,2, \ldots, N)$ are dummy variables and $N$ is size of sample.

Now simulate $N$ paths of the market prices of $n$ assets and set up a suitable linear problem on those simulated paths is given by

$$
\begin{aligned}
& \underset{w}{\max _{w}} E\left(R_{T}^{w}\right), \\
& \text { subject to } \\
& \qquad R_{T, k}^{w}=\sum_{i=1}^{n} w_{i} R_{T, k}^{i}, \quad k=1,2, \ldots, N, \\
& \quad \sum_{i=1}^{n} w_{i}=1, \\
& w_{i} \geq 0, \quad i=1,2, \ldots, n, \\
& \quad\left(\alpha+\frac{1}{N(1-\beta)} \sum_{k=1}^{N} z_{k}\right) \leq d, \\
& R_{T, k}^{w}+\alpha+z_{k} \geq 0, \quad k=1,2, \ldots, N, \\
& z_{k} \geq 0, \quad k=1,2, \ldots, N .
\end{aligned}
$$

Here note that dimension of the problem is of the order of the number of simulated paths $N$. However, this also shows that the number of simulation runs 
determines the size of the problem as considering more investment opportunities would only slightly increase the dimension of the problem (infact, each further asset leads to just one more variable).

Now assume that structured products $S_{1}, S_{2}, \ldots, S_{m}$ mature at time $t_{1} \leq$ $t_{2} \leq \cdots \leq t_{m}=T$ respectively where $t_{j} \leq T, j=1, \ldots, m-1$. Here $T$ is investor's horizon time. So each structured product (except the last one) of the portfolio matures before investor's horizon time $T$. Now considering to allocate the portfolio over investment horizon $T$, the investor needs to take in account how the payoff of the products that mature before the investment horizon $T$ should be reinvested. The presence of such intermediate payments is the main extension of the work done by Martellini [4]. So modified multi-period investment model is given as

$$
\begin{aligned}
& \max _{w} E\left(R_{T}^{w, x}\right), \\
& \text { subject to } \\
& \qquad R_{T, k}^{w, k}=\sum_{i=1}^{u} w^{i} R_{T, k}^{i}+\sum_{j=1}^{m} w_{u+j} R_{T, k}^{u+j, x^{i}}, \quad k=1,2, \ldots, N, \\
& 1+R_{T, k}^{u+j, x}=\left(1+\prod_{k}^{j}\right)\left[x_{j+1}^{j}\left(1+r_{T-t_{j}, k}^{j+1}\right)+\cdots+x_{n}^{j}\left(1+r_{T-t_{j}, k}^{m}\right)\right], \\
& 1+R_{T, k}^{u+j, x^{j}}=\left(1+\prod_{k}^{j}\right) \sum_{\ell=j+1}^{n} x_{\ell}^{j}\left(1+r_{T-t_{j}, k}^{\ell}\right), \quad k=1,2, \ldots, N, \\
& \sum_{i=1}^{n} w_{i}=1, \quad j, \ldots, 1, \ldots, \\
& w_{i} \geq 0, \quad i=1,2, \ldots, n, \\
& R_{T, k}^{w, x^{j}}+\alpha+z_{k} \geq 0, \quad k=1,2, \ldots, N, \\
& \left(\alpha+\frac{1}{N(1-\beta)} \sum_{k=1}^{N} z_{k}\right) \leq d, \\
& \quad k=1,2, \ldots, N, \\
& z_{k} \geq 0, \quad \sum_{\ell=j+1}^{n} x_{\ell}^{i}, \quad j=1,2, \ldots, m-1,
\end{aligned}
$$




$$
x_{\ell}^{j} \geq 0, \quad \ell=j+1, \ldots, n, j=1,2, \ldots, m-1,
$$

where $x_{\ell}^{j}$ is the percentage of the structured product $S_{j}$ 's payoff that is allocated to asset $\ell$ after maturity at time $t_{j}, r_{T-t_{j}, k}^{\ell}$ is the return of asset $\ell$ for the remaining time $T-t_{j}, \prod_{k}^{j}$ is the return of asset $S_{j}$ from the start of the investment period until its maturity and $k$ corresponds to scenario. Here we assume that each bond matures at $T$ and payoff of each structured product is reinvestment in the remaining structured products using weight vector $x^{j}=\left(x_{j+1}^{j}, \ldots, x_{n}^{j}\right)$. Only last structured product $S_{m}$ matures at horizon time $T$. This problem is not linear optimization problem. One can not solve it with simplex method only. But it can be solved by creating optimization loops. Each intermediate re-balancing point creates a new loop. So $(m-1)$ outer loops are needed. We assume that $x^{j}$ 's be the weight vectors that maximize the expected return for the particular product $S_{j}$. Thus reducing the whole problem to solve $m$ linear optimization problems. Firstly, solve optimization problem with maximizing expected return as target for the period $t_{m-1}$ to $t_{m}=T$ to attain $x^{m-1}$, $r_{T-t_{m-1}, k}^{m-1}$ and thus complete the return vector for product $S_{m-1}$. Repeating the process until the full return vectors of all $(m-1)$ products are known. At the end solve the well-defined optimization problem to attain vector $w$.

\section{Conclusion}

The results indicate that the portfolio having structured products can be superb investment for investors searching for low risk alternatives with a limited downside. The paper suggests to allocate the portfolio to maximize expected return attaining as much capital-protection as possible. CVaR risk measure is used because it is both subadditive and choerent risk measure. Also CVaR risk measure is adequate to use in scenario based optimization. This set up is more relevant for an investor that must re-balance its portfolio at different intermediate time points and especially when the investor is allowed to invest in more than one structured products. This approach can handle large number of instruments and scenarios.DOI:http://dx.doi.org/ 10.2139/ssrn.267256.

\section{References}

[1] C. Acerbi, Spectral measures of risk: A coherent representation of subjective risk aversion, Journal of Banking and Finance, 26(7) (2002), 15051518. DOI: $10.1016 / \mathrm{s} 0378-4266(02) 00281-9$ 
[2] P. Artzner, F. Delboen, J.M. Eber and D. Heath, Coherent measures of risk, Mathematical Finance, 9(3) (1999), 203-228. DOI: 10.4236/jmf.2013.33039

[3] H. Markowitz, Portfolio selection, Journal of Finance, 7(1) (1952), 77-91. DOI:10.1111/j.1540/6261.1952tb01525.x.

[4] L. Martellini, K. Simsek, K. Goltz, Structured forms of investment strategies in institutional investor's portfolios, Technical Report, EDHEC Risk and Asset Management Research Centre, 2005.

[5] R.T. Rockafellar and P. Uryasev, Conditional Value-at-Risk for general loss distributions, ISE Department, Working papers 20015DOI:http://dx.doi.org/ 10.2139/ssrn.267256

[6] R. Korn and S. Zeytun, Solving optimal investment problems with structured products under CVaR constraints, Optimization, 58 (3) (2009), 291304. DOI: $10.1080 / 0233 / 930902741739$

[7] R.T. Rockafellar and S. Uryasev, Optimization of conditional Value-atRisk, Journal of Risk, 2(3) (2000). DOI: 10.1.1.132.1960 